\title{
Performance Measurement Parameter Selection of PHM System for Armored Vehicles Based on Entropy Weight Ideal Point
}

\author{
Yuanhong Liu \\ School of Equipment Engineering, Armed Police Force Engineering University, Xi'an, 710000 \\ China \\ qingyun0611@sina.com
} Keywords: Armored Vehicle; PHM Performance Measurement; Entropy Weight; Ideal Point;
Evaluation Index

\begin{abstract}
A performance measurement parameter selection method of PHM (Prognostics and Health Management) system for armored vehicle based on entropy weight ideal point is proposed, aiming at the problem that there are too many performance measurement parameters of armor vehicle PHM system and lack of theoretical guidance to the selection of these parameters. a measurement parameter set of PHM system, according to the relationship among armored vehicle safety comprehensive plan, training use, maintenance, equipment management requirements and PHM system performance measurement parameters. Then, the armored vehicle PHM system requirements are used to working as evaluation indexes of PHM performance measurement parameters, and an objective attribute matrix is constructed, based on which the entropy weight of the evaluation index is calculated, and then the ideal points of optimal membership matrix is calculated. Then, the close degrees between evaluation parameters and ideal points are calculated, and the performance measurement parameter sequence is obtained according to the value of close degrees. The results of the example shows the order of performance measurement parameters can be obtained effectively through the index superiority matrix without expert weight, which provides basis for performance measurement parameter selection of PHM system.
\end{abstract}

\section{Introduction}

With the rapid development of information technology and automation technology, technical integration and complexity of armored vehicles are getting higher and higher. The problem of online maintenance and security in the process of warfare and training, such as difficult and incomplete state information collection, inefficient troubleshooting and rapid increase, is becoming more and more serious. How to ensure the overall equipment performance has become a hot research topic. Prognostic and health management technology comes into being, and continues to grow and develop. PHM system has been in the field of defense and industrial applications, and shows its huge potential for development and application prospects [1].

In the development and promotion process of PHM technology and system, equipment demand side has two main functions [2]: the first is to meet the needs of tactical technical indicators to provide development direction for industrial sector, and the second is to verify and confirm the performance of PHM system to ensure that products meet the design requirements. Therefore, to develop PHM technology and PHM system, the first problem to be solved is how to evaluate PHM system performance scientifically.

The performance measurement system of PHM is shown in Figure 1 [3-5], mainly divided into test measurements, fault diagnosis measurements, fault prediction measurements, calculation measurements and cost efficiency measurements. For different application objects, the PHM system performance measurement parameters are also different [6-10]. In practical PHM performance of armored vehicle, measurement parameters are of many types, and it is impractical to adopt all of them. So the performance measurement parameters of PHM system must be optimized to determine a set of appropriate, scientific parameters. 


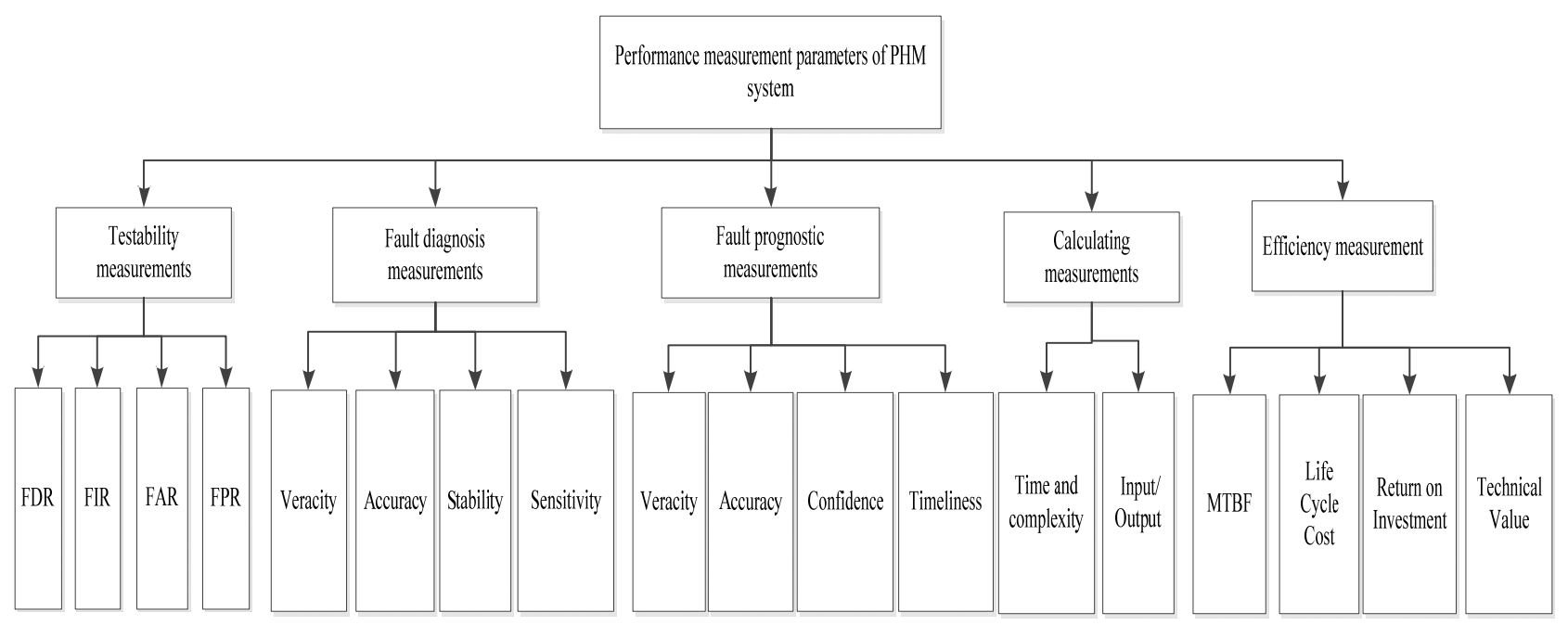

Figure 1. Performance measurement parameters of PHM system

This paper presents a selection method of armored vehicle PHM system performance measurements based on entropy weight ideal point. Combining tactical and technical indicators of military users with PHM implementation technology, this paper puts forward a set of performance measurement parameters suitable for armored vehicle PHM system, which provides theoretical support to demonstration, effective evaluation and verification of armored vehicle PHM system...

\section{Evaluation Index of PHM System Performance Measurement Parameters in Armored Vehicles}

For a given armored vehicle, there are generally more comprehensive requirements of protection, troop training and use, maintenance plan and equipment group management. Armored Vehicle Support Integrated Program Users are responsible for planning and implementing the necessary resources for acquisition, transfer, and maintenance to maintain equipment operation. The needs of the integrated program users are mainly reflected in reducing repair recovery time, reducing maintenance support equipment and personnel, reducing workload, reducing periodic inspections and reducing inventory of spare parts. The corresponding PHM technology is mainly for the diagnosis, prediction, the corresponding PHM performance indicators include testability measurement, fault diagnosis, fault prediction metrics. The goal of training user is to provide process safety information, to reduce future operation and instruction uncertainty. The demand is mainly reflected in the minimum false alarm rate, and the corresponding PHM system performance indicators include false alarm rate and so on.

The bottom line of maintenance user is to minimize repair duplication, the same as re-service equipment as soon as possible. The goal of maintenance users is mainly reflected in the rapid fault location and isolation, including reduction of non-reproducible and retentive faults, accurate fault location, maximum fault coverage, reduced failure, reduced PHM system maintenance time and so on. The corresponding PHM system performance metrics include fault detection rate, fault isolation rate, fault diagnosis metrics, fault prediction metrics and so on.

Armored vehicle group management user is defined as a decision-making manager for influencing life extension, operating costs and future planning. The group management maximizes equipment availability and mission success while minimizing cost and resource usage, including extending service hours, reducing unplanned maintenance and operating costs. The corresponding PHM system performance metrics include fault prediction metrics, life cycle costs, and so on. Therefore, according to the above requirements, the relationship between armored vehicle PHM system performance indicators and use requirements is shown in Table 1 . In the performance measurement parameter selection of armored vehicle PHM system, the armored vehicle comprehensive support plan, the force training and use, maintenance and equipment group management user needs are used as evaluation indexes by the entropy right ideal point method. 
Table 1. Relationship between User Requirements of Armored Vehicle and PHM System Performance Measurement Parameters

\begin{tabular}{|c|c|c|c|c|c|c|c|c|c|c|c|c|c|c|c|c|c|c|}
\hline $\begin{array}{l}\text { Parameters User } \\
\text { requirements }\end{array}$ & $\begin{array}{c}\mathrm{FD} \\
\mathrm{R} \\
\end{array}$ & \begin{tabular}{l|l}
$\mathrm{FI}$ \\
$\mathrm{R}$ \\
\end{tabular} & $\begin{array}{c}\mathrm{FA} \\
\mathrm{R}\end{array}$ & $\begin{array}{c}\mathrm{FP} \\
\mathrm{R}\end{array}$ & $\begin{array}{c}\text { FD } \\
\mathrm{V}\end{array}$ & $\begin{array}{c}\mathrm{FD} \\
\mathrm{A}\end{array}$ & $\begin{array}{c}\text { FDS } \\
\mathrm{e}\end{array}$ & \begin{tabular}{|c} 
FDS \\
$\mathrm{t}$
\end{tabular} & $\begin{array}{l}\text { FP } \\
\text { V }\end{array}$ & \begin{tabular}{c|}
$\mathrm{FP}$ \\
$\mathrm{A}$
\end{tabular} & $\begin{array}{l}\mathrm{FP} \\
\mathrm{C}\end{array}$ & \begin{tabular}{c|}
$\mathrm{FP}$ \\
$\mathrm{T}$
\end{tabular} & $\begin{array}{l}\text { CT } \\
\text { C }\end{array}$ & $\begin{array}{l}\mathrm{I} / \\
\mathrm{O}\end{array}$ & \begin{tabular}{|c|} 
MTB \\
F
\end{tabular} & $\begin{array}{c}\text { LF } \\
\text { C }\end{array}$ & $\begin{array}{c}\mathrm{RO} \\
\mathrm{I}\end{array}$ & $\begin{array}{l}\mathrm{T} \\
\mathrm{V}\end{array}$ \\
\hline Reduced repair time & $\sqrt{ }$ & $\sqrt{ }$ & $\sqrt{ }$ & & $\sqrt{ }$ & $\sqrt{ }$ & $\sqrt{ }$ & $\sqrt{ }$ & & & & & & & & & & \\
\hline Reduced ground support & $\sqrt{ }$ & $\sqrt{ }$ & $\sqrt{ }$ & $\sqrt{ }$ & $\sqrt{ }$ & $\sqrt{ }$ & $\sqrt{ }$ & $\sqrt{ }$ & $\sqrt{ }$ & $\sqrt{ }$ & $\sqrt{ }$ & $\sqrt{ }$ & & & & & & \\
\hline Reduced workload & $\sqrt{ }$ & $\sqrt{ }$ & $\sqrt{ }$ & $\sqrt{ }$ & $\sqrt{ }$ & $\sqrt{ }$ & $\sqrt{ }$ & $\sqrt{ }$ & $\sqrt{ }$ & $\sqrt{ }$ & $\sqrt{ }$ & $\sqrt{ }$ & & & & & & \\
\hline Reduced periodic check & & & & $\sqrt{ }$ & & & & & $\sqrt{ }$ & $\sqrt{ }$ & $\sqrt{ }$ & $\sqrt{ }$ & & & & & & \\
\hline maximized component life & & & & $\sqrt{ }$ & & & & & $\sqrt{ }$ & $\sqrt{ }$ & $\sqrt{ }$ & $\sqrt{ }$ & & & & & & \\
\hline Optimized inventory & $\sqrt{ }$ & $\sqrt{ }$ & & $\sqrt{ }$ & $\sqrt{ }$ & $\sqrt{ }$ & $\sqrt{ }$ & $\sqrt{ }$ & $\sqrt{ }$ & $\sqrt{ }$ & $\sqrt{ }$ & $\sqrt{ }$ & & & & & & \\
\hline Reduced FAR & & & & & & & & & & & & & & & & & & \\
\hline Reduced non-recurrence & $\sqrt{ }$ & $\sqrt{ }$ & & $\sqrt{ }$ & $\sqrt{ }$ & $\sqrt{ }$ & $\sqrt{ }$ & $\sqrt{ }$ & $\sqrt{ }$ & $\sqrt{ }$ & $\sqrt{ }$ & $\sqrt{ }$ & & & & & & \\
\hline Accurate fault location & $\sqrt{ }$ & $\sqrt{ }$ & & & & & & & & & & & & & & & & \\
\hline Increased FDR & $\sqrt{ }$ & & & & & & & & & & & & & & & & & \\
\hline Reduced failure & & & & $\sqrt{ }$ & & & & & $\sqrt{ }$ & $\sqrt{ }$ & $\sqrt{ }$ & $\sqrt{ }$ & & & & & & \\
\hline $\begin{array}{l}\text { Reduced repair time of } \\
\text { PHM system }\end{array}$ & & & & & & & & & & & & & & & $\sqrt{ }$ & & & \\
\hline Extended service time & & & & $\sqrt{ }$ & & & & & $\sqrt{ }$ & $\sqrt{ }$ & $\sqrt{ }$ & $\sqrt{ }$ & & & & & & \\
\hline $\begin{array}{l}\text { Reduced unplanned } \\
\text { maintenance }\end{array}$ & & & & $\sqrt{ }$ & & & & & $\sqrt{ }$ & $\sqrt{ }$ & $\sqrt{ }$ & $\sqrt{ }$ & & & & & & \\
\hline Reduced operating cost & & & & & & & & & & & & & $\sqrt{ }$ & $\sqrt{ }$ & $\sqrt{ }$ & $\sqrt{ }$ & $\sqrt{ }$ & $\sqrt{ }$ \\
\hline
\end{tabular}

FDR-Fault Detection Rate, FIR-Fault Isolation Rate, FAR-Fault Alarm Rate, FPR-Fault Prognostic Rate, FDV-Fault Detection Veracity, FDA-Fault Detection Accuracy, FDSe-Fault Detection Sensitivity, FDSt-Fault Detection Stability, FPV-Fault Prognostic Veracity, FPC-Fault Prognostic Confidence, FPT-Fault Prognostic Timeliness, CTC-Calculating Time and Complexity, I/O- Input and Output, MTBF-Mean Time Between Failure, LFC-Life Cycle Cost, ROI-Return On Investment, TV -Technical Value

\section{Evaluation Model of Entropy Right Ideal Point}

Supposing there are $n$ test parameters to be evaluated, each parameter is described by $m$ evaluation indicators, the index eigenvalue matrix of $n$ test parameters is $X=\left(x_{i j}\right)_{m \times n}(i=1,2, \cdots, m, j=1,2, \cdots n)$, which can be normalized to obtain a relative superior Membership matrix $R=\left(r_{i j}\right)_{m \times n}$. For the larger $x_{i j}$, the better the parameters of evaluation index, the calculation of relative superiority is as follows.

$$
r_{i j}=\frac{x_{i j}-\min \left(x_{i j}\right)}{\max \left(x_{i j}\right)-\min \left(x_{i j}\right)}
$$

For the smaller $x_{i j}$, the better the parameters of evaluation index, the calculation method of relative merit is as follows.

$$
r_{i j}=\frac{\max \left(x_{i j}\right)-x_{i j}}{\max \left(x_{i j}\right)-\min \left(x_{i j}\right)}
$$

In the formula (2), $\max \left(x_{i j}\right)$ and $\min \left(x_{i j}\right)$ are maximum value and minimum value of the index $x_{i j}$ for different parameters under the same indicators. According to the concept of traditional entropy, the entropy of the index can be defined as follows.

$$
H_{i}=\frac{\sum_{j=1}^{m} f_{i j} \ln f_{i j}}{\ln n}, f_{i j}==\frac{r_{i j}}{\sum_{j=1}^{n} r_{i j}}
$$

For $r_{i j}=0$, the $\ln f_{i j}$ makes no sense. In order to make sense, not contrary to the meaning of 
entropy, the amendment is made as follows.

$$
f_{i j}=\frac{1+r_{i j}}{\sum_{j=1}^{n}\left(1+r_{i j}\right)}
$$

Therefore, the entropy weight $X S_{i}$ of the i-th evaluation index is defined as follows.

$$
X S_{i}=\frac{1-H_{i}}{\sum_{j=1}^{m}\left(1-H_{j}\right)}
$$

After considering the entropy weight, the attribute matrix $P$ is as follows:

$$
P=\left[\begin{array}{ccc}
p_{11} & \cdots & p_{1 n} \\
\vdots & & \vdots \\
p_{m 1} & \cdots & p_{m n}
\end{array}\right]=\left[\begin{array}{ccc}
X S_{1} r_{11} & \cdots & X S_{1} r_{1 n} \\
\vdots & & \vdots \\
X S_{m} r_{m 1} & \cdots & X S_{m} r_{m n}
\end{array}\right]
$$

For ideal point $P^{*}=\left(P_{1}^{*}, P_{2}^{*}, \cdots, P_{m}^{*}\right)$, where $P_{m}^{*}$ is the maximum value of each row, that is, the optimal value, the proximity of the evaluated parameter to the ideal point is as follows.

$$
T_{j}=1-\frac{\sum_{i=1}^{m} p_{i j} P_{i}^{*}}{\sum_{i=1}^{m} P_{i}^{* 2}}, j=(1,2, \cdots, n)
$$

In the formula(7), $T_{j} \in[0,1]$, the smaller the degree of close $T_{j}$, the better the rated parameters. According to the calculated value $T_{j}$, the pros and cons. order of the parameters can be obtained in accordance with the order from small to large to evaluate the parameters.

\section{Example}

From the above analysis, the evaluation matrix $X$ of the PHM performance indexes of armored vehicles is composed of 15 evaluation indexes and 18 parameters to be evaluated. The element value $x_{i j}$ in the matrix is evaluated using the fuzzy numbers 1 to 9 . The higher $x_{i j}$, the higher the correlation between the evaluation index and the evaluated parameters, the greater the correlation between the PHM technical requirements of the armored vehicle and the PHM performance parameters. If the evaluation index is not related to the evaluation parameters, the $x_{i j}$ is 0 , and the PHM performance measurement parameters evaluation matrix is shown in table 2 . When the $x_{i j}$ is larger, that is, the higher the technical requirements of the armored vehicle PHM, the higher the corresponding requirements of the evaluation parameters. The relative superiority matrix is obtained by choosing the benefit-based normalization method to normalize the evaluation table. The process of calculating relative merit function is based on each fuzzy value. When the parameter is not related to other indexes, the corresponding relative merit is the deterministic value 0 , that is, irrelevant. 
Table 2. Measurement Parameters Evaluation Matrix of Armored Vehicle PHM System Performance

\begin{tabular}{|c|c|c|c|c|c|c|c|c|c|c|c|c|c|c|c|c|c|c|}
\hline $\begin{array}{c}\text { Parameters } \\
\text { User requirements }\end{array}$ & F & FIR & AR & PR & DV & FDA & FDs & FDSt & PY & FPA & FPC & FPT & CTC & $\mathrm{I} / \mathrm{O}$ & MTBF & $\mathrm{LFC}$ & $\mathrm{ROI}$ & \\
\hline Reduced repair time & 5 & 5 & 5 & 0 & 4 & 2 & 3 & 3 & 0 & 0 & 0 & 0 & 0 & 0 & 0 & 0 & 0 & \\
\hline $\begin{array}{l}\text { Reduced ground } \\
\text { support }\end{array}$ & 4 & 5 & 4 & 4 & 4 & 2 & 2 & 3 & 3 & 2 & 2 & 2 & 0 & 0 & 0 & 0 & 0 & 0 \\
\hline Reduced workload & 3 & 4 & 4 & 3 & 3 & 2 & 2 & 2 & 3 & 2 & 2 & 2 & 0 & 0 & 0 & 0 & 0 & \\
\hline $\begin{array}{r}\text { Reduce } \\
\mathrm{ch}\end{array}$ & 0 & 0 & 0 & 4 & 0 & 0 & 0 & 0 & 3 & 2 & 2 & 3 & 0 & 0 & 0 & 0 & 0 & 0 \\
\hline $\begin{array}{r}\text { maximized } \\
\text { li }\end{array}$ & 0 & 0 & 0 & 5 & 0 & 0 & 0 & 0 & 5 & 3 & 2 & 4 & 0 & 0 & 0 & 0 & 0 & 0 \\
\hline Optimized & 5 & 5 & 0 & & 4 & 2 & 2 & 3 & 4 & & 2 & 3 & & 0 & & 0 & 0 & 0 \\
\hline & 0 & 0 & $g$ & 0 & & c & & & & & ( & 0 & & & & & & \\
\hline $\begin{array}{r}\text { Redu } \\
\text { rec }\end{array}$ & 5 & 5 & 0 & 3 & 4 & 2 & 3 & 3 & 4 & 2 & 3 & 3 & 0 & 0 & 0 & 0 & 0 & 0 \\
\hline Accurate & 6 & 9 & 0 & 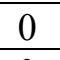 & & 0 & & & & & 0 & & & & & & & \\
\hline & 9 & 0 & c & 0 & & 0 & 0 & 0 & 0 & 0 & 0 & 0 & & 0 & & & 0 & 0 \\
\hline Redu & 0 & 0 & 0 & 5 & & 0 & & & 5 & 3 & 3 & 5 & & 0 & & & 0 & 0 \\
\hline $\begin{array}{r}\text { Reduced } \\
\text { PHI }\end{array}$ & 0 & 0 & 0 & 0 & 0 & 0 & 0 & 0 & 0 & 0 & 0 & 0 & 0 & 0 & 9 & 0 & 0 & 0 \\
\hline Extende & 0 & 0 & 0 & 3 & & 0 & & 0 & 4 & 2 & 3 & $T$ & 0 & 0 & $\sigma$ & 0 & 0 & 0 \\
\hline Reduce & 0 & 0 & 0 & 5 & 0 & 0 & 0 & 0 & 5 & 3 & 3 & 5 & 0 & 0 & 0 & 0 & 0 & 0 \\
\hline $\begin{array}{l}\text { Reduced operating } \\
\text { cost }\end{array}$ & 0 & 0 & 0 & 0 & 0 & 0 & 0 & 0 & 0 & 0 & 0 & 0 & 2 & 1 & 3 & 6 & 3 & 4 \\
\hline
\end{tabular}

First, the entropy $H$ is as follows according to the formulas (3) and (4).

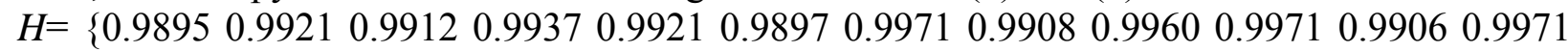
$0.99320 .99060 .9951\}$

Then the entropy weight XS of the evaluation index is as follows according to Eq. (5).

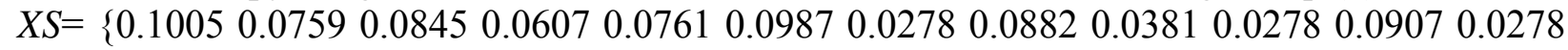
$0.06550 .09070 .0469\}$

According to the formula (6), we obtain the ideal point $\mathrm{P}^{*}$ as follows based on the index attribute matrix after considering the entropy weight.

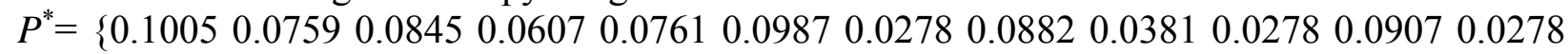
$0.06550 .09070 .0469\}$

The degree of close $T$ between the evaluation object and $\mathrm{P}^{*}$ can be calculated according to the formula (7).

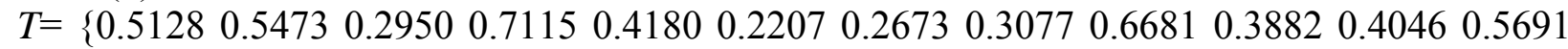
$0.00960 .00480 .02440 .02870 .01430 .0191\}$

According to the calculated $\mathrm{T}$ value, the test parameters obtained by the priority order are shown in table 3 .

Table 3. Test Parameter Priorities

\begin{tabular}{|c|c|c|c|c|c|c|}
\hline Parameters & FDR & FIR & FAR & FPR & FDV & FDA \\
\hline Order & 5 & 4 & 10 & 1 & 6 & 11 \\
\hline Parameters & FDSe & FDSt & FPV & FPA & FPC & FPT \\
\hline Order & 12 & 9 & 2 & 8 & 7 & 3 \\
\hline Parameters & CTC & SI/O & MTBF & LFC & ROI & TV \\
\hline Order & 17 & 18 & 14 & 13 & 16 & 15 \\
\hline
\end{tabular}

Therefore, the PHM system performance measurement parameters and indicators of armor vehicle which should be determined are FPR, FPA, FPV, FPT, FIR, FDR, FDV, FPC, FPA etc. according to the need to give priority. These parameters are concerned directly to system reliability, maintainability, which should also be given priority in practical application. 


\section{Conclusion}

(1) A method of selecting the performance parameters of armored vehicle PHM system based on ideal point of entropy is proposed, and a scientific, reasonable and streamlined PHM system performance parameter set is selected.

(2) In the absence of expert weight, the entropy weight ideal point method determines the index weight according to the evaluation index characteristic value of PHM system performance parameter, which reduces the method of obtaining evaluation index by expert scoring or reconstructing judgment matrix Weighting process, and the three-tier multi-objective evaluation model is simplified to a twolevel target evaluation model.

\section{References}

[1]. F. Feng, A. Si, W. Xing, et al. Application and development of fault prediction and health management technology. Journal of Armored Force Engineering, vol. 23, pp. 1-6, 2009.

[2]. F. Feng. Research on the composition and evaluation method of armored vehicle fault prediction and health management system index system. General Equipment Department, 2015.

[3]. B. Jing, X. Jiao, Y. Huang. A PHM System Performance Measurement Architecture Based on User and Function. Journal of Electronic Measurement and Instrumentation, vol. 29, pp. 157-164, 2015

[4]. B. Leao, T. Yoneyama, G. Rocha. Progenitor's performance metrics and their relation to requirements, design, verification and cost-benefit. International Conference on PHM, 2008.

[5]. B. Jing, Z. Yang, J. Zhang, et al. Prediction and verification of fault prediction and health management system. Computer Engineering and Applications, vol. 47, pp. 23-27, 2011

[6]. A. Wang, Y. Zheng, A. Wang, et al. The selection and evaluation of health management index system of complex weapon system. Computer Measurement and Control, vol. 22, pp. 1169-1171, 2014

[7]. S. Miao, Y. Yang, X. Lei, et al. Research on Performance Measurement System of Military Aircraft Forecasting and Health Management System TIAN Jian. Measurement and Control Technology, vol. 30, pp. 106-110, 2011.

[8]. Z. Zeng, Z. Ren, Y. Wu. Research on indexes and verification technology of airborne PHM system. Prognostics \& System Health Management Conference, 2010.

[9]. Z. Yang, B. Jing, R. Zhang. Airborne system fault prediction and health management verification and evaluation method. Measurement and Control Technology, vol. 31, pp. 101-104, 2012

[10]. Z. Wei, F. Liu, L. Chen. Aero - engine health management user's diagnostic predictive index system. Measurement and Control Technology, vol. 38, pp. 27-35, 2012 